\title{
Queueing Systems With Four Different Main Server's Interruptions and a Stand-By Server
}

\author{
Rehab F. Khalaf ${ }^{1}$ \\ ${ }^{1}$ Jaber Alali, Alahmadi, Kuwait \\ Correspondence: Rehab F. Khalaf, Jaber Alali, Alahmadi, Kuwait. E-mail: rihabfadil@gmail.com \\ Received: October 7, 2013 Accepted: December 21, 2013 Online Published: January 6, 2014 \\ doi:10.5539/ijsp.v3n1p49 URL: http://dx.doi.org/10.5539/ijsp.v3n1p49
}

\begin{abstract}
This work studied a queuing system with four different main server interruptions and a stand-by server replaces the main server during any potential stop. The main server has five statues in this system where it is either services the customers( active) or it does not work because of one of the four reason; original vacation, extended vacation, it is broken-down but the repairs did not start yet (delay time) and broken-down servers under repairs. The arrivals are assumed to attend the system in batches. All the distributions of the service, vacation, extended vacation, repairs and delay times all follow general distributions while the breakdown times and the stand-by service times are exponentially distributed.
\end{abstract}

Keywords: server vacation, extended vacation, server breakdown, repair delay, stand-by sever

\section{Introduction}

Queueing systems with server interruptions have been studied by many authors. In his survey (Doshi, 1986) mentioned more than one kind of server interruptions but he named all of them "vacations". Years later the researchers in queueing systems classified these interruptions according to their reasons as vacations, breakdowns and delay. Some researchers studied queueing systems with server vacation. In their work, Choi and Kim (2003) considered a two-phase queueing system with server vacations and Bernoulli feedback, where the server takes vacation if the system becomes empty at the moment of the completion of the second phase services. Madan and Abu Al-Rub (2004) assumed that there is one server in the system, the server may have a single vacation policy with elective phase type server vacations. Arumuganathan and Jeyakumar (2005) Assumed that the server may leave to have vacation whenever the number of waiting customers in the queue does not exceed certain Number ' $N$ ', When the server returns from a vacation and if the queue length is still less than ' $N$ ', he leaves for another vacation and so on.

Several authors studied queuing systems with server breakdowns. We can cite Aissani and Artalejo (1998), Wang, Cao, and Li (2001), Madan, Abu-Dayyah, and Gharaibeh (2003), Wang (2004) and Senthil and Arumuganathan (2010). Queueing systems with vacations and breakdowns, have been studied by other researchers e.g., Maraghi, Madan, and Dowman (2010) and M. Jain and A. Jain (2010).

In recent years, Khalaf, Madan, and Lucas (2011a, 2011b) introduced two new kinds of server interruptions; the extended vacation and the delay time. Khalaf et al. (2011c, 2012) studied the queuing system already developed by them in their previous work but this time they assumed that the system equipped with a stand-by server works when system major server is on vacation or under repairs.

In this work a queueing system studied with all the four different server stops; the server could be on vacation, extended vacation, it is broken-down but the repairs did not start yet (delay time) or it is broken-down under repairs. The main adventure of this work is that it is assumed that system equipped with a stand- by server works during all the main server 4 stops. We present a clear closed form of the most interested performing measures (the mean number of customers in the system, the mean number of customers in the queue and the mean waiting time in the system).

It is considered that the customers arrive to the system in batches, the vacations are Bernoulli scheduled, it is optional that the server goes to extended vacations, all the distributions of the service, vacation, extended vacation, 
repairs and delay times are assumed to follow different general distributions while the breakdown times and the stand-by service times follow two different exponential distributions.

Although tens of published queueing systems are special cases of the system studied in this paper, only four particular cases are mentioned in the last section.

\section{Model Assumptions}

The queueing system $M^{[X]} / G / 1$ has been studied in this work. It is known that there is only one server in this system, we will call it the "main server" because there is a stand- by server services the customers when the main server cannot provide service to the customers.

We consider that the customers reach the system in groups of size $i, i=1,2,3, \ldots$. The mean arrival rate of the groups is $\lambda>0$. The first order probability that a group of $i$ customers arrives at the system during a short interval of time $(t, t+\Delta t)$ is given by $\lambda c_{i} \Delta t$, where $0 \leq c_{i} \leq 1$ and $\sum_{i=1}^{\infty} c_{i}=1$.

The service provide to the groups according to their arrival order, where all the customers of the first arrival group served before the customers of the second arrival group and so on. The groups provided service one by one on a first come- first served basis. Inside the group customers provide the service randomly where all the customers in the first group should receive their service before the customers in the second group and so on.

The service times of the main server follow a general (arbitrary) distribution with distribution function $G(s)$ and density function $g(s)$. Let $\mu(x) \Delta x$ be the conditional probability of service completion during the interval $(x, x+\Delta x]$, given that the elapsed service time is $x$, so that $\mu(x)=\frac{g(x)}{1-G(x)}$ and, therefore $g(s)=\mu(s) e^{-\int_{0}^{s} \mu(x) d x}$.

The main server may have four deferent interruptions; the first interruption is the original vacation. It is assumed that the vacation time is Bernoulli scheduled, when the main server completes any service it may take vacation (with probability $p$ ) or continue providing service (with probability $1-p$ ). The server vacation times are generally distributed with distribution function and density function $B(v), b(v)$ respectively. Let $\beta(x) \Delta x$ denotes the conditional probability that the server completes a vacation period during the interval $(x, x+\Delta x]$ given that the elapsed vacation time is $x$, so that $\beta(x)=\frac{b(x)}{1-B(x)}$ and, therefore $b(v)=\beta(v) e^{-\int_{0}^{v} \beta(x) d x}$.

The second potential stop of the main server is the extended vacation. On completion of a vacation period, the server has an option of taking an extended vacation. The server may take an extended vacation with probability $r(0 \leq r \leq 1)$ or it may rejoin the system immediately after completion of the original vacation with probability $1-r$.

The extended vacation times are generally distributed. The distribution function of the extended vacation times is $W(x)$. The density function of the extended vacation times is $w(x)$. Let $\varphi(x) \Delta x$ denotes the conditional probability that the server completes extended vacation period during the interval $(x, x+\Delta x]$ given that the elapsed time of the extended vacation time is $x$, so that $\varphi(x)=w(x) /(1-W(x))$ and therefore, $w(x)=\varphi(x) \exp \left(-\int_{0}^{x} \varphi(t) d t\right)$.

The server may encounter random breakdowns, the breakdown times follow Poisson distribution with mean breakdown rate $\alpha>0$. Additional we assume that when the server breaks down, the customer whose service is interrupted comes back to the head of the queue but it is instantly taken up for service by the stand-by server.

Once the system breakdown, the repairs do not start immediately and there is a delay time waiting for repairs to start, this is the third possible server stop. The delay times are generally distributed with delay distribution function and delay density function $F(x), f(x)$ respectively. Let $\theta(x) \Delta x$ denotes the conditional probability that the server finishes a delay period during the interval $(x, x+\Delta x]$ given that the elapsed delay time is $x$, so that, $\theta(x)=f(x) /(1-F(x))$ and therefore, $f(x)=\theta(x) \exp \left(-\int_{0}^{x} \theta(t) d t\right)$.

When the server breaks down the repair process may start any time, where this is the fourth potential server stops. The repair times are generally distributed with repairs distribution function and repairs density function $H(x), h(x)$ respectively. Let $\gamma(x) \Delta x$ denotes the conditional probability that the repairs of the server completed during the interval $(x, x+\Delta x]$ given that the elapsed repair time is $x$, so that $\gamma(x)=h(x) /(1-H(x))$ implies $h(x)=\gamma(x) \exp \left(-\int_{0}^{x} \gamma(t) d t\right)$.

When the main server is on vacation, extended vacation, waiting for repairs to start or under repair, in all these four deferent interruptions, there is a stand-by server servers the customers until the main server returns. The stand-by service times are exponentially distributed with stand-by service rate $\delta>0$ and mean stand-by service time $1 / \delta$.

When the main server rejoins the system after any interruption, the customer being served by the stand-by server 
transferred to the main server to start a service over. All stochastic processes involved in the system are independent of each other.

\section{Notations and Definitions}

Let's consider the following definitions and notations:

(1) Probability that at time $t$, there are $n(n \geq 0)$ customers in the queue excluding the customer in service and the elapsed service time of this customer is $x$ is denoted by $P_{n}(t, x)$. The probability that at time $t$, there are $n(n \geq 0)$ customers in the queue excluding the customer in service irrespective of the value of $x$ is denoted by $P_{n}(t)=\int_{0}^{\infty} P_{n}(t, x) d x$.

(2) Probability that at time $t$, there are $n(n \geq 0)$ customers in the queue and the server is on original vacation with elapsed original vacation time $x$ is denotes by $V_{n}(t, x) . V_{n}(t)=\int_{0}^{\infty} V_{n}(t, x) d x$ denotes the probability that at time $t$, the number of customers in the queue is $n(n \geq 0)$ and the server is on vacation whatever the value of $x$ is. The probability generating function of the queue size when the server is on vacation is given by $V_{q}(x, z)=\sum_{n=0}^{\infty} z^{n} V_{n}(x)$, $V_{q}(z)=\sum_{n=0}^{\infty} z^{n} V_{n}$.

(3) $R_{n}(t, x)$ denotes the probability that there are $n(n \geq 0)$ customers in the queue at time $t$, while the server is on repair process with elapsed repair time $x . R_{n}(t)=\int_{0}^{\infty} R_{n}(t, x) d x$ refers to probability that there are $n(n \geq 0)$ customers in the queue at time $t$, while the server is on repair process whatever the value of $x$ is. The probability generating function of the queue size when the server is under repair is given by $R_{q}(x, z)=\sum_{n=0}^{\infty} z^{n} R_{n}(x), R_{q}(z)=$ $\sum_{n=0}^{\infty} z^{n} R_{n}$.

(4) $Q(t)$ is the probability that a58889+t time $t$, there are no customers in the system and the server is idle but available in the system. $\lim _{t \rightarrow \infty} Q(t)=Q$ is the steady state probability corresponding to $Q(t)$.

(5) $D_{n}(t, x)$ denotes the probability that, there are $n(n \geq 0)$ customers waiting in the queue at time $t$, and the server does not serve because it is suffering from breakdown and it is waiting for repairs to start with elapsed delay time $x . D_{n}(t)=\int_{0}^{\infty} D_{n}(t, x) d x$ refers to the probability that there are $n(n \geq 0)$ customers in the queue at time $t$, and the server is waiting for repairs to start whatever the value of $x$ is. The probability generating function of the queue size when the server is waiting for a repair to start (on delay) is given by $D_{q}(x, z)=\sum_{n=0}^{\infty} z^{n} D_{n}(x), D_{q}(z)=\sum_{n=0}^{\infty} z^{n} D_{n}$.

(6) $E_{n}(t, x)$ denotes the probability that at time $t$ there are $n(n \geq 0)$ customers waiting in the queue, and the server is on an extended vacation with elapsed extended vacation time $x$.

(7) The probability that at time $t$, there are $n(n \geq 0)$ customers waiting the queue while the server is on an extended vacation whatever the value of $x$ is $E_{n}(t)=\int_{0}^{\infty} E_{n}(t, x) d x$. The probability generating function of the queue size when the server is on an extended vacation is given by $E_{q}(x, z)=\sum_{n=0}^{\infty} z^{n} E_{n}(x), E_{q}(z)=\sum_{n=0}^{\infty} z^{n} E_{n}$.

(8) $C(z)=\sum_{n=1}^{\infty} z^{n} c_{n}$ is probability generating function of the batch arrival size.

(9) $A_{n}(x)=\lim _{t \rightarrow \infty} A_{n}(t, x)$ is the steady state probability corresponding to $A_{n}(t, x) . A_{n}=\lim _{t \rightarrow \infty} A_{n}(t)$ is the steady state probability corresponding to $A_{n}(t)$, where $A=P, V, D, E, R$.

\section{The Equations Represent the System}

The following set of equations characterize the model described in Section 2

$$
\begin{gathered}
\frac{\partial}{\partial x} P_{n}(x)=-(\lambda+\mu(x)+\alpha) P_{n}(x)+\lambda \sum_{i=1}^{n-1} c_{i} P_{n-i}(x), n \geq 1 \\
\frac{\partial}{\partial x} P_{0}(x)=-(\lambda+\mu(x)+\alpha) P_{0}(x) \\
\frac{\partial}{\partial x} V_{n}(x)=-(\lambda+\beta(x)+\delta) V_{n}(x)+\lambda \sum_{i=1}^{n} c_{i} V_{n-i}(x)+\delta V_{n+1}(x), n \geq 1 \\
\frac{\partial}{\partial x} V_{0}(x)=-(\lambda+\beta(x)+\delta) V_{0}(x)+\delta V_{1}(x) \\
\frac{\partial}{\partial x} E_{n}(x)+(\lambda+\theta(x)+\delta) E_{n}(x)=\lambda \sum_{i=1}^{n} c_{i} E_{n-i}(x)+\delta E_{n+1}(x), n \geq 1
\end{gathered}
$$




$$
\begin{gathered}
\frac{\partial}{\partial x} E_{0}(x)=-(\lambda+\theta(x)+\delta) E_{0}(x)+\delta E_{1}(x) \\
\frac{\partial R_{n}(x)}{\partial x}=-(\lambda+\gamma(x)+\delta) R_{n}(x)+\lambda \sum_{i=1}^{n} c_{i} R_{n-i}(x)+\delta R_{n+1}(x), n \geq 1 \\
\frac{\partial R_{0}(x)}{\partial x}=0 \\
\frac{\partial}{\partial x} D_{n}(x)+(\lambda+\phi(x)+\delta) D_{n}(x)=\lambda \sum_{i=1}^{n} c_{i} D_{n-i}(x)+\delta R_{n+1}(x), n \geq 1 \\
\frac{\partial R_{0}(x)}{\partial x}=0 \\
\lambda Q=\int_{0}^{\infty} R_{0}(x) \gamma(x) d x+(1-p) \int_{0}^{\infty} P_{0}(x) \mu(x) d x+(1-r) \int_{0}^{\infty} V_{0}(x) \beta(x) d x+\int_{0}^{\infty} E_{0}(x) \theta(x) d x
\end{gathered}
$$

A full explanation of how to get Equations (1), (2) and (11) is given in (Khalaf, 2012). The same argument can be considered to explain Equations (3) to (10).

The next boundary condition will be used to solve the set of equations represent the system.

$$
\begin{gathered}
P_{n}(0)=(1-p) \int_{0}^{\infty} P_{n+1}(x) \mu(x) d x+(1-r) \int_{0}^{\infty} V_{n+1}(x) \beta(x) d x \\
+\int_{0}^{\infty} E_{n+1}(x) \theta(x) d x+\int_{0}^{\infty} R_{n+1}(x) \gamma(x) d x+\lambda c_{n+1} Q, n \geq 0 \\
V_{n}(0)=p \int_{0}^{\infty} P_{n}(x) \mu(x) d x, n \geq 0 \\
E_{n}(0)=r \int_{0}^{\infty} V_{n}(x) \beta(x) d x, n \geq 0 \\
R_{n}(0)=\int_{0}^{\infty} D_{n}(x) \varphi(x) d x, n \geq 0 \\
D_{n}(0)=\alpha \int_{0}^{\infty} P_{n-1}(x) d x=\alpha P_{n-1}, n \geq 1 \\
D_{0}(0)=0
\end{gathered}
$$

Where the boundary condition (12) has been explained in (Khalaf, 2012). The same argument can be considered to explain the other boundary conditions.

\section{Queue Size Distribution at a Random Epoch}

The main aim in this section is to find the probability generating function (PGF) of the queue size at a random epoch. Let $S_{q}(z)$ denote the PGF of the queue size irrespective of the state of the system. Thus

$$
S_{q}(z)=P_{q}(z)+V_{q}(z)+E_{q}(z)+D_{q}(z)+R_{q}(z)
$$

To get $P_{q}(z), V_{q}(z), E_{q}(z), D_{q}(z)$, and $R_{q}(z)$ the method used in (Khalaf et al., 2011, 2012) to get

$$
\begin{gathered}
P_{q}(z)=\frac{-Q m\left(1-G^{*}(a)\right)}{a\left(z-G^{*}(a)\left(1-p+p B^{*}(\omega) k\right)\right)-\alpha z J} \\
V_{q}(z)=\frac{-a Q m p G^{*}(a)\left(1-B^{*}(\omega)\right)}{\omega a\left(z-G^{*}(a)\left(1-p+p B^{*}(\omega) k\right)\right)-\alpha z \omega J} \\
E_{q}(z)=\frac{-a Q m r p G^{*}(a) B^{*}(\omega)\left(1-W^{*}(\omega)\right)}{a \omega\left(z-G^{*}(a)\left(1-p+p B^{*}(\omega) k\right)\right)-\alpha z \omega J} \\
D_{q}(z)=\frac{-Q m \alpha z\left(1-G^{*}(a)\right)\left(1-F^{*}(\omega)\right)}{a \omega\left(z-G^{*}(a)\left(1-p+p B^{*}(\omega) k\right)\right)-\alpha z \omega J}
\end{gathered}
$$


Where $a=\lambda-\lambda C(z)+\alpha, \omega=\lambda-\lambda C(z)+\delta-\frac{\delta}{z}, J=\left(1-G^{*}(a)\right) F^{*}(\omega) H^{*}(\omega), m=\lambda-\lambda C(z)$, and $k=1-r+r W^{*}(\omega)$. $G^{*}(a), B^{*}(a), W^{*}(a), F^{*}(a), H^{*}(a)$ are the Laplace-Stieltjes transform of the service time $G(x)$, vacation time $B(x)$, extended vacation time $W(x)$, delay time $F(x)$ and repairs time $H(x)$ respectively.

Adding equations from (18) to (21) we get

$$
S_{q}(z)=\frac{-Q m\left(\left(1-G^{*}(a)\right)\left[\omega+\alpha z\left(1-F^{*}(\omega) H^{*}(\omega)\right)\right]+a p G^{*}(a)\left(1-B^{*}(\omega) k\right)\right)}{a \omega\left(z-G^{*}(a)\left(1-p+p B^{*}(\omega) k\right)\right)-\alpha z \omega J}
$$

To find $Q$, we use the normalization condition, $S_{q}(1)+Q=1$.

The value of $S_{q}(1)$ is indeterminate, so we apply L'Hopital's rule two times on Equation (22).

To find the steady state average queue length $L_{q}$, the known relation $L_{q}=\left.\frac{d}{d z} S_{q}(z)\right|_{z=1}$ could be used. We can derive the mean waiting time in the queue by using Little's law $W_{q}=L_{q} / \lambda$.

\section{Particular Cases}

The queueing system studied in this work can be considered as a very general system. A lot of systems studied by the same author (Rehab F. Khalaf) or by other others can be a special cases of this system. In this section we will discuss three particular cases as following:

\subsection{No Stand by Server}

If the service system is not equipped with a stand by server then $\delta=0, \omega=\lambda-\lambda C(z)+\delta-\frac{\delta}{z}=\lambda-\lambda C(z)=m$, from Equation (22) we obtain

$$
S_{q}(z)=\frac{-Q\left(\left(1-G^{*}(a)\right)\left(m+\alpha z\left(1-F^{*}(m) H^{*}(m)\right)\right)+a p G^{*}(a)\left(1-B^{*}(\omega) k\right)\right)}{a\left(z-G^{*}(a)\left(1-p+p B^{*}(m) k\right)\right)-\alpha z J}
$$

The result given in Equation (23) agrees with results given in (Khalaf, 2012).

\subsection{No Delay Starting the Repairs, No Extended Vacation Times and No Stand by Server}

If we assume there is no stand by server, no extended vacation and no delay this means that $\delta=0, W^{*}(w)=1$, $r=0$, and $F^{*}(w)=1$, respectively. Then we get

$$
S_{q}(z)=\frac{-Q\left(\left(1-G^{*}(a)\right)\left[m+\alpha z\left(1-H^{*}(\omega)\right)\right]+a p G^{*}(a)\left[1-B^{*}(m)\right]\right)}{a\left(z-G^{*}(a)\left(1-p+p B^{*}(m)\right)\right)-\alpha z\left(1-G^{*}(a)\right) H^{*}(m)}
$$

The result given in Equation (24) are the same results given by (Maraghi et al., 2010).

\subsection{No Vacation, No Extended Vacation, Stand by Server and No Breakdown ( No Delay, No Repairs)}

If we assume there is no vacation, no extended vacation, no stand by server and no breakdown (no delay, no repairs).

This means that $p=0, W^{*}(w)=1, r=0, \delta=0, \alpha=0, F^{*}(w)=1$ and $F^{*}(w)=1$, respectively. From Equation (22), we get

$$
S_{q}(z)=\frac{-Q\left(1-G^{*}(a)\right)}{\left(z-G^{*}(a)\right)}=\frac{Q m\left(G^{*}(a)-1\right)}{\left(z-G^{*}(a)\right)}
$$

This result agree with the known results of $M^{[X]} / G / 1$ given by (Bunday, 1996).

\section{Conclusion}

We have studied $M^{[X]} / G / 1$ queueing system with four different types of the main server interruptions. These interruptions are: vacation, extended vacation, random server break-down and repairs delay. It is assumed that there is a stand-by server ready to serve the customers during the main server interruptions. The service times, vacation times, extended vacation times, repair times and delay times, all follow general distributions. This work presents a clear closed form of the most interested performing measures.

\section{References}

Aissani, A., \& Artalejo, J. R. (1998). On the single server retrial queue subject to breakdowns. Queueing Systems, 30(3-4), 309-321. http://dx.doi.org/10.1023/A:1019125323347

Arumuganathan, R., \& Jeyakumar, S. (2005). Steady state analysis of a bulk queue with multiple vacations, setup times with N-policy and closedown times. Applied Mathematical Modelling, 29, 927-986. http://dx.doi.org/10.1016/j.apm.2005.02.013 
Bunday, B. D. (1996). An introduction to queueing theory. Oxford, England: Oxford University Press.

Choi, D., \& Kim, T. (2003). Analysis of a two-phase queueing system with vacations and Bernoulli feedback. Stochastic Analysis and Applications, 21(5), 1009-1019. http://dx.doi.org/10.1081/SAP120024702

Doshi, B. T. (1986). Queueing systems with vacations-a survey. Queueing Systems, 1, 29-66. http://dx.doi.org/10.1007/BF01149327

Jain, M., \& Jain, A. (2010). Working vacation queueing model with multiple types of server breakdowns. Applied Mathematical Modelling, 34, 1-13. http://dx.doi.org/10.1016/j.apm.2009.03.019

Khalaf, R. F. (2012). On Some Queueing Systems with Server Vacations, Extended Vacations, Breakdowns, Delayed Repairs and Stand-bys. (A PhD theses submitted to School of Information Systems, Computing and Mathematics, Brunel University.)

Khalaf, R. F., Madan, K. C., \& Lucas, C. A. (2011a). An $M^{[X]} / G / 1$ Queue with Bernoulli Schedule, General Vacation Times, Random Breakdowns, General Delay Times and General Repair Times. Applied mathematical Sciences, 5(1), 35-51.

Khalaf, R. F., Madan, K. C., \& Lucas, C. A. (2011b). An $M^{[X]} / G / 1$ Queue with Bernoulli Schedule General Vacation Times, General Extended Vacations, Random Breakdowns, General Delay Times for Repairs to Start and General Repair Times. Journal of Mathematics Research, 3(4), 8-20. http://dx.doi.org/10.5539/jmr.v3n4p8

Khalaf, R. F., Madan, K. C., \& Lucas, C. A. (2011c). On a Batch Arrival Queuing System Equipped with a Stand-by Server During Vacation Periods or the Repairs Times of the Main Server. Journal of Probability and Statistics, Article ID 812726. http://dx.doi.org/10.1155/2011/812726

Khalaf, R. F., Madan, K. C., \& Lucas, C. A. (2012). On an $M^{[X]} / G / 1$ Queueing System with Random Breakdowns, Server Vacations, Delay Times and a Standby. International Journal of Operational Research, 15(1), 30-47. http://dx.doi.org/10.1504/IJOR.2012.048290

Madan, K. C., \& Abu Al-Rub, A. Z. (2004). On a single server queue with optional phase type server vacations based on exhaustive deterministic service and a single vacation policy. Applied Mathematics and Computation, 149, 723-734.

Madan, K. C., Abu-Dayyeh, W., \& Gharaibeh, M. (2003). On two parallel servers with random breakdowns. Soochow Journal of Mathematics, 29(4), 413-423.

Maraghi, F. A., Madan, K. C., \& Darby-Dowman, K. (2010). Bernoulli schedule vacation queue with batch arrivals and random system breakdowns having general repair time distribution. International Journal of Operational Research, 7(2), 240-256. http://dx.doi.org/10.1504/IJOR.2010.030805

Senthil, M., \& Arumuganathan, R. (2010). An $M^{[X]} / G / 1$ retrial queue with two-phase service subject to active server breakdowns and two types of repair. International Journal of Operational Research, 8(3), 261-291. http://dx.doi.org/10.1504/IJOR.2010.033540

Wang, J. (2004). An $M / G / 1$ queue with second optional service and server breakdown. Computers and Mathematics with Applications, 47, 1713-1723. http://dx.doi.org/10.1016/j.camwa.2004.06.024

Wang, J., Cao, J., \& Li, Q. (2001). Reliability analysis of the retrial queue with server breakdowns and repairs. Queueing Systems, 38(4), 363-380. http://dx.doi.org/10.1023/A:1010918926884

\section{Copyrights}

Copyright for this article is retained by the author(s), with first publication rights granted to the journal.

This is an open-access article distributed under the terms and conditions of the Creative Commons Attribution license (http://creativecommons.org/licenses/by/3.0/). 